\title{
Desarrollo del pensamiento bistórico. Análisis de exámenes de los estudiantes
}

Development of the Historic Thought. Analysis of Tests for Students

Artículo de investigación | Research Article

Fecha de recepción: 07 de febrero de 2019

Fecha de aceptación: 05 de junio de 2019

Fecha de disponibilidad en línea: marzo de 2020

doi: 10.11144/Javeriana.m13.dpha

Nancy Palacios-Mena n.palaciosm@uniandes.edu.co Universidad de los Andes, Colombia

ORCID: http://orcid.org/0000-0003-1318-0728

\section{Luz Yehimy Chaves-Contreras} yehimy1927@gmail.com

Secretaria de Educación de Bogotá, Colombia D ORCID: http://orcid.org/0000-0001-8133-2645

William Alfonso Martin-Moreno wmartin82@hotmail.com

Secretaria de Educación de Bogotá, Colombia

ORCID: http://orcid.org/0000-0002-2223-9085

Para citar este artículo | To cite this article Palacios-Mena, N., Chaves-Contreras, L. Y. \& Martin-Moreno, W. A. (2020). Desarrollo del pensamiento histórico. Análisis de exámenes de los estudiantes. magis, Revista Internacional de Investigación en Educación, 13, 1-29. doi: 10.11144/Javeriana.m13.dpha 


\section{Resumen}

El objetivo principal de este artículo es analizar el contenido y el nivel cognitivo en exámenes de historia de estudiantes de trece colegios públicos de Bogotá. Se utilizó un diseño metodológico mixto, que combinó la sistematización de datos cualitativos y cuantitativos del contenido de los exámenes. Los resultados indican que la enseñanza y la evaluación tienen como eje un tiempo lineal, que gira en torno a las épocas. En gran parte de los exámenes, se solicita a los estudiantes principalmente memorizar y recordar datos, por tanto se trata de una evaluación elemental que privilegia los contenidos conceptuales e involucra poco los procedimentales.

\section{Palabras clave}

Aprendizaje; educación; enseñanza de la historia; evaluación de la educación; examen

\section{Abstract}

The main objective in this article is to analyze the contents and cognitive level in the history tests for students in 13 public schools in Bogotá. A mixed methodological design was used; it combined the systematization of both qualitative and quantitative data from the test contents. The results indicate that the backbone for the teaching and evaluation is a linear timeline divided into eras. To a greater extent, the tests ask the students basically to memorize and recall data. This is an elementary evaluation that prioritizes the conceptual contents and involves quite little procedural contents.

\section{Keywords}

Learning; education; history education; educational evaluation; examinations 
Descripción del artículo | Article description

Este artículo es uno de los productos de la investigación

El aprendizaje de las ciencias sociales y el desarrollo de

competencias en primaria y secundaria.

\section{Introducción}

Este artículo es uno de los productos de una investigación que tiene como objetivo determinar los niveles de desarrollo de habilidades alcanzadas por los estudiantes colombianos de primaria y secundaria en el área de ciencias sociales, a través del análisis de los resultados de la Prueba Saber $11^{\circ}$ y de sus productos de trabajo en clase, como cuadernos, guías, narrativas y exámenes.

El texto tiene como propósito fundamental determinar el contenido y las habilidades evaluadas en exámenes de ciencias sociales de estudiantes de educación básica y media en colegios públicos de Bogotá.

El artículo es pertinente porque al debate sobre la necesidad de comprender la evaluación como un proceso que debe aportar a la mejora de la enseñanza y el aprendizaje (Merchán, 2005) se suma el cuestionamiento de autores como Souto \& Claudino (2009), Sáiz (2013) y Souto, Fuster \& Sáiz (2014), según el cual la evaluación se ha convertido en el certificado de ciertos estándares de calidad que acaban condicionando las prácticas pedagógicas, la escogencia de los contenidos y los exámenes que los docentes hacen a sus estudiantes.

Este artículo pretende aportar al campo de la didáctica de las ciencias sociales, más concretamente a la didáctica de la historia. Los trabajos de Castillo (2004), Ravela (2010), Rojas (2014), Seixas \& Morton (2013), Cooper (2013), Souto, Fuster \& Saíz (2014), Prado \& Medina (2014) y Pérez (2015) han identificado que las fortalezas y debilidades que se evidencian en las tareas que los estudiantes realizan en clase se reflejan en los desempeños que obtienen en los exámenes que hacen los profesores y en las pruebas de ingreso a la universidad. Estos autores coinciden en que los procesos de evaluación tienen que estar acompañados de trabajos en el aula más retadores y desafiantes para los estudiantes. Por lo tanto, este artículo invita a una lectura dialogada entre las actividades de aprendizaje del alumnado y las evaluaciones internas y externas, con miras a la realización de un mejor seguimiento a los aprendizajes de los estudiantes y a la construcción de nuevos enfoques teóricos y metodológicos para orientar el trabajo en el aula. 
Desde la postura de Rincón (2008), si bien los niños y jóvenes muestran actitudes favorables a la enseñanza de la historia, tienen muchas dificultades en el desarrollo de actividades de aprendizaje en las que se les exigen habilidades conceptuales y procedimentales complejas. Las conclusiones de estos estudios coinciden también en que un trabajo alternativo al que usualmente se realiza en las clases, direccionado al desarrollo de habilidades y no a la reproducción de contenidos, contribuye a que los estudiantes alcancen mayores desarrollos cognitivos, construyan conocimientos más significativos e incrementen su capacidad comunicativa (interpretación, argumentación y proposición) en el área de ciencias sociales.

Así pues, este trabajo se alinea con otros, como el realizado por Gómez \& Miralles (2013), que se han ocupado de indagar por la evaluación, al considerarla "un factor esencial debido a la relación que hay entre el modo de evaluar y la metodología didáctica" (p. 93). Para Gómez \& Miralles (2013), "debemos diagnosticar la forma en la que se evalúan los conocimientos de ciencias sociales para saber cómo se enseñan esos contenidos, y si éstos responden realmente a un aprendizaje por competencias" (p. 93). En consecuencia, el eje central de este artículo es el análisis de las habilidades y los conocimientos que se evalúan en los exámenes de ciencias sociales, información fundamental para determinar fortalezas y oportunidades de mejora en los procesos de enseñanza y aprendizaje.

\section{Marco teórico}

\section{Desarrollo del pensamiento histórico y evaluación}

Los investigadores que vienen haciendo estudios sobre el desarrollo del pensamiento histórico - la perspectiva didáctica más prolífera en la enseñanza de la disciplina en las últimas décadas - han dedicado gran parte de su reflexión a lo que aprenden y lo que no los estudiantes en primaria y secundaria. Para Gómez-Carrasco, Miralles-Martínez \& Chapman (2017),

dirigir la educación hacia la consecución de competencias educativas básicas exige un cambio en la enseñanza y la práctica en el aula, que conlleva también una reflexión sobre la naturaleza de la evaluación del alumnado y los métodos e instrumentos utilizados para la misma (p. 182).

De esta manera, la enseñanza y evaluación son dos procesos que se entrelazan e intercambian en el proceso de aprendizaje de los estudiantes. Investigaciones recientes que han suscrito su marco teórico al campo del desarrollo del pensamiento histórico vienen señalando que tanto en las actividades de aprendizaje como en los procesos de evaluación de la clase 
de historia es pertinente la incorporación de conocimientos de primer y de segundo orden, "los primeros se refieren a los conceptos de lo que trata la historia, el contenido. El segundo tipo aporta herramientas necesarias para el estudio del pasado y la construcción de explicaciones históricas" (Gómez \& Miralles, 2017, p. 62). Así,

en las clases de historia el desarrollo de habilidades debe concentrarse en contenidos estratégicos de segundo orden, a través de habilidades como: a) relevancia histórica, que implica la capacidad de interrogarnos sobre qué y quién del pasado vale la pena ser recordado y estudiado b) fomentar el análisis y la obtención de evidencias a partir de fuentes y pruebas históricas c) fomentar entre los alumnos el desarrollo de la conciencia histórica, entendida como la capacidad para interrelacionar fenómenos del pasado y del presente d) la habilidad de construir o representar las narrativas del pasado, es decir, comunicar de forma oral o escrita, relatos o explicaciones argumentadas sobre el pasado (Gómez \& Miralles, 2017, pp. 63 y 64).

En suma, para Gómez \& Miralles (2017), el desarrollo de capacidades intelectuales superiores en los estudiantes, como análisis, síntesis, conceptualización, manejo de la información, pensamiento sistémico, pensamiento crítico, investigación y metacognición, son capacidades presentes en la enseñanza y evaluación del pensamiento histórico a través de los conocimientos históricos de segundo orden.

En esta misma línea, Domínguez (2015) propone desarrollar competencias del pensamiento histórico a través de los conceptos sustantivos y conceptos metodológicos o de segundo orden en historia. Este autor afirma que

los conceptos sustantivos se refieren a la sustancia del contenido histórico. Los segundos se refieren a cómo se construye dicho conocimiento del pasado, por los rasgos metodológicos principales de la historia como disciplina: fuentes y pruebas, causas y consecuencias, relatos e interpretaciones, relevancia, progreso, decadencia (p. 46).

Para Domínguez (2015), el trabajo con fuentes históricas en clase favorece la adquisición de destrezas o técnicas de trabajo que son útiles para familiarizar al alumno con el manejo de la información. Pero, además, "permiten el desarrollo de destrezas como: reflexión sobre los conceptos de prueba y fuente, destrezas historiográficas de tratamiento de las fuentes, destrezas de comprensión lectora y comunicación" (p. 87). Otro de los rasgos característicos del trabajo de aula propuesto por el autor son las 
explicaciones causales que ponen en juego las destrezas cognitivas, ya que es un ejercicio complejo para el estudiante, pues "exige contemplar a la vez distintos factores o causas, evaluar el papel e importancia de cada una de ellas y ordenar la exposición (generalmente escrita) de una manera más clara y equilibrada" (Domínguez, 2015, p. 136).

De esta manera, desde la postura de Domínguez (2015), en la enseñanza de la historia necesariamente se tienen que involucrar habilidades como: obtener información explícita, obtener información por inferencia, integrar, interpretar, sintetizar, escribir y argumentar.

\section{Enseñanza de la historia y desarrollo de habilidades cognitivas}

Uno de los debates contemporáneos sobre la enseñanza de las ciencias sociales ha girado en torno a la adopción de un enfoque interdisciplinar o de uno que privilegie la enseñanza de las disciplinas. Quienes abogan por el primer enfoque defienden la necesidad de dar a los estudiantes herramientas que les permitan comprender el pasado y el presente de la sociedad, incluyendo los aportes de la sociología, la antropología y la ciencia política, y no exclusivamente los aportes de la geografía y de la historia (Asensio, Carretero \& Pozo, 1986; Guimera \& Carretero, 1992). Quienes abogan por el segundo enfoque, y principalmente quienes defienden la enseñanza de la historia, enfatizan en la "necesidad [de] que los estudiantes comprendan los procesos de cambio en el tiempo histórico y su influencia en el momento presente; es decir, que aprendan a pensar históricamente" (Carretero \& Montanero, 2008, p. 135).

Entre los autores que han centrado sus estudios en el desarrollo del pensamiento histórico —una de las líneas que defiende el enfoque disciplinar-, hay consenso desde hace ya un tiempo sobre la importancia de posicionar una enseñanza de la historia que aporte a la formación política y ciudadana. Desde esta perspectiva, el objetivo principal de la enseñanza de la disciplina es que el alumnado apropie conocimientos, habilidades y actitudes útiles para la comprensión del mundo en el que viven y el devenir de las sociedades en épocas anteriores (Carretero \& Montanero, 2008; Carretero \& Voss, 2004).

Así pues, las investigaciones que se han venido publicando en la línea del desarrollo del pensamiento histórico han concluido que es fundamental desplazarse de una enseñanza de la historia centrada en personajes, fechas y eventos a otra centrada en promover la capacidad de comprender el tiempo histórico, razonar causalmente y valorar e interpretar críticamente las fuentes de información histórica (Carretero \& Montanero, 2008; Lucero \& Montanero, 2008; Montanero, 2001). 
Autores de esta línea de pensamiento, como Shemilt (1983) y Carretero \& Limón (1993), coinciden en que la historia es una forma de conocimiento con unas lógicas, métodos y perspectivas sobre las que se debe dar instrucción en la escuela, sin que esto implique formar a los estudiantes de primaria y secundaria para ser historiadores. Este tipo de instrucción plantea la pertinencia de hacer cambios significativos en las estrategias pedagógicas y didácticas implementadas en los salones de clase. Trabajos realizados en España, Brasil, Chile, Norteamérica y el Reino Unido han evidenciado que esta nueva enseñanza de la historia requiere que el alumnado alcance un pensamiento formal y desarrolle habilidades cognitivas avanzadas (Carretero, 1993; Pozo y Carretero, 1989).

Desde la perspectiva de Fuster (2017) y Sáiz \& López-Facal (2014), los avances que se han producido en los estudios sobre el desarrollo del pensamiento histórico en la educación básica y media han recibido una notable influencia de los hallazgos de las investigaciones en la epistemología de la historia y la psicología evolutiva. Para Éthier, Demers \& Lefrançois (2010), los progresos en la comprensión de lo que los alumnos aprenden o no en la clase de historia se deben en gran medida a los avances del desarrollo cognitivo, de tal forma que "los avances que en este campo reflejan los cambios en la concepción de la forma en que los alumnos se apropian de instrumentos cognitivos tal como el pensamiento histórico" (p. 65).

Según Domínguez (2015), el diálogo fructífero entre los didactas de la historia y los psicólogos cognitivos ha sido fundamental en la progresiva internacionalización de las investigaciones sobre la enseñanza de la primera, "las dos líneas de investigación, didáctica y psicología, corroboran el papel clave que tienen los conceptos metodológicos en el aprendizaje del pensamiento histórico" (p. 48). Domínguez también destaca que los trabajos evolucionaron de una perspectiva piagetiana hacia enfoques basados en la comparación entre novatos y expertos, de tal forma que en la actualidad han aumentado de manera considerable los estudios con un enfoque cognitivo que comparan el desempeño de estudiantes e historiadores en tareas concretas, como el manejo de fuentes o la construcción de narrativas históricas.

Estos estudios mencionados coinciden en señalar que los estudiantes y futuros maestros participantes en las investigaciones han evidenciado deficiencias en el razonamiento de conocimientos propios de las ciencias sociales y, más concretamente, en conocimientos de historia en la mayor parte de las tareas que se realizan en dichas clases, pues dicho alumnado no alcanza a aplicar conceptos o procedimientos ni a formular explicaciones elaboradas y acabadas de los hechos, y se quedan en la reproducción de información de un lugar en otro (Plá, 2012; Prats, 2016; Sáiz, 2013, 2014; Valls \& López-Facal, 2011). 
Carretero, Pozo \& Asensio (1989) son reiterativos en que las dificultades de comprensión y las de ejecución de tareas complejas se deben a que los estudiantes no han alcanzado altos niveles en su desarrollo cognitivo. Desde la postura de estos autores,

el desarrollo cognitivo no es solamente un conjunto de estrategias de razonamiento que pueden aplicarse a cualquier contenido, sino que también consiste en paquetes de información específica, que dependen de la experiencia concreta de cada alumno y que se debería adquirir mediante la instrucción escolar (p. 24).

Para Carretero, Pozo \& Asencio (1989), es muy oportuno preguntar si los alumnos no resuelven las tareas escolares de ciencias sociales y de historia porque no han adquirido las operaciones formales o porque no reciben en la escuela la estimulación y la formación suficiente para que mejoren su desarrollo cognitivo.

En esta misma línea, Santisteban (2010), Martineau (1999) y Pagès (2009) subrayan que el aprendizaje de la historia debe pasar por procesos de enseñanza que integren los fundamentos pedagógicos y cognitivos de la disciplina con la manera de razonar, con sus propios procedimientos, sus conceptos e interpretaciones y con la incorporación de perspectivas globales y locales que combinen elementos estáticos y dinámicos, de cambio y continuidad que busquen relaciones entre el pasado, el presente y el futuro.

\section{Los exámenes y la evaluación de la clase de historia}

Es innegable la centralidad que tiene hoy la evaluación en el sistema educativo. Como han señalado Gómez, Miralles \& Molina (2015), esto puede obedecer a la importancia que se viene dando a las evaluaciones nacionales y a las internacionales. En el caso colombiano, tanto en la legislación educativa que se ha ocupado exclusivamente del tema (como el Decreto 230 de 2002 y el 1290 de 2009) como en la que se ha dado recomendaciones curriculares y metodológicas, los lineamientos y los estándares curriculares, los derechos básicos de aprendizaje y las mallas de aprendizaje tienen una mención importante, además de indicar que el proceso de evaluación debe aportar información sobre qué y cómo aprenden los estudiantes, según los documentos de legislación educativa que ha publicado el Ministerio de Educación Nacional de Colombia. Dicho proceso también debe ser útil para mejorar la enseñanza y el aprendizaje.

Por otro lado, pese a las críticas de distintos sectores de la academia y sindicatos de profesores a la decisión del país de adoptar una educación por competencias, el discurso sobre la implementación de este enfoque 
educativo se volvió usual en el profesorado colombiano de la educación básica y media, y las competencias pasaron a ser parte de las clases y de la labor diaria de los docentes. Según Miralles, Molina \& Santisteban (2011), Santos (1993) y Alfageme \& Miralles (2009), la inclusión de la enseñanza y la evaluación por competencias se ha dado paralelamente a la consolidación de sistemas de evaluación que han establecido mecanismos y procedimientos para recoger información conceptual, procedimental y actitudinal. Así pues, como indican Gómez et al. (2015),

la aparición en la década del dos mil del nuevo concepto pedagógico de competencias supuso un nuevo reto e impulso para el concepto de evaluación educativa. La necesidad de educar en competencias, con un alto nivel de contenidos procedimentales, y de aplicar conocimientos interdisciplinares, destrezas y actitudes a situaciones determinadas, debía obligar a una mayor presencia de la evaluación formativa en dicho proceso educativo (p. 10).

Desde la perspectiva de Mainer (2002), Gómez et al. (2015) y Monteagudo, Molina \& Miralles (2015), la importancia que sigue teniendo el examen en los procesos de evaluación de la enseñanza de la historia se debe a que los procedimientos y criterios de dicho proceso continúan ligados a finalidades culturalistas de la historia y a que factores como el número de estudiantes, la necesidad de establecer criterios objetivos y fáciles de defender ante posibles reclamaciones determina el tipo de capacidades evaluadas en las pruebas, lo que limita el desarrollo de aquellas competencias intelectuales que pueden favorecer el desarrollo del pensamiento histórico.

Las investigaciones de López (1997), Trepat (2012), Calatayud (2000), Gómez \& Miralles (2013), Monteagudo, Villa \& Miralles (2015) y Gómez et al. (2015) subrayan que el examen sigue siendo el instrumento de evaluación más usado y que el proceso de evaluación valora principalmente el aprendizaje de conocimientos conceptuales. Para Pellegrino-Chudowski \& Glaser (2001, citado por Gómez et al., 2015),

cada evaluación independientemente del propósito, debe apoyarse en tres pilares: un modelo teórico de cómo los estudiantes representan el conocimiento y desarrollan competencias en el ámbito temático en el que se les evalúa; las tareas o situaciones que permiten observar el desempeño de los estudiantes; y un método de interpretación para hacer inferencias a partir de pruebas de rendimiento (p. 10).

La aplicación de pruebas o exámenes en los que se indaga por fechas, lugares y personajes siguen estando presentes en la historia escolar; los 
exámenes siguen siendo los elementos más utilizados para valorar los conocimientos adquiridos por los estudiantes. La aplicación sistemática y generalizada de exámenes en la clase de historia, dejando de lado otras formas de evaluar el conocimiento, puede conducir a desatender un principio de la educación actual que señala que no se trata de medir lo que se sabe, sino lo que se sabe hacer con el conocimiento que se tiene (VanSledright, 2014).

Estudios en la enseñanza de la historia en los que se ha utilizado exámenes (Merchán, 2009; Molina \& Calderón, 2009; Monteagudo \& Vera, 2017; Vera, Moreno \& Torres, 2014) han subrayado que persisten concepciones del proceso de evaluación como una acción para calificar y no como una acción que permite realizar acciones conducentes a mejorar los aprendizajes de los estudiantes y los procesos de enseñanza, aunque esto último abunde en la retórica del profesorado.

Así pues, los exámenes siguen teniendo el primer lugar como instrumento de evaluación o en sentido estricto de calificación del alumnado, si bien, como han señalado Pérez (1995) y Monteagudo \& Vera (2017), no se trata de exterminarlos de la escuela, porque siguen teniendo utilidad e importancia, parece fundamental que no sigan siendo los principales indicadores para determinar la promoción y reprobación de los estudiantes de un grado a otro y de la calidad de la educación que se ofrece en la escuela primaria y secundaria.

Según Plá \& Pagès (2014), si bien es cierto que la producción académica sobre desarrollo del pensamiento histórico en América Latina viene en aumento en las últimas décadas, dicho aumento no es similar en todos los países. Colombia, Argentina y Brasil tienen el mayor número de trabajos, seguidos por Chile y México, pero en países como Uruguay, Perú y Nicaragua hay solo unos pocos trabajos. Las diferencias en la cantidad de investigaciones se explican por el poco apoyo económico a investigaciones en las áreas de ciencias sociales y educación, por la escasez de trabajos interdisciplinarios y por la cantidad de programas de posgrados en historia y educación.

Los estudios publicados se han centrado en el análisis de los materiales de trabajo, como cuadernos y libros de texto; las prácticas pedagógicas y didácticas del profesorado; el lugar de la historia en las ciencias sociales; los currículos de historia; la formación de conciencia histórica en los estudiantes, y las percepciones que estos tienen de la enseñanza de la historia y los profesores (Plá \& Pagès, 2014).

El trabajo de Plá \& Pérez (2013), con participantes de diferentes edades, sobre aspectos de la historia reciente de México a través de la fotografía como fuente histórica, concluyó que había un deficiente desarrollo en el análisis de fuentes y de la capacidad de diferenciar el ejercicio analítico objetivo de las valoraciones personales y subjetivas sobre la historia 
reciente. Las conclusiones de Plá \& Pérez (2013) muestran una pluralidad en las formas de pensar el pasado y de analizar las fuentes, pues en ocasiones una mejor competencia narrativa o explicación histórica no necesariamente está directamente relacionada con un mejor análisis de la imagen. Plá \& Pérez (2013) también concluyen que las reformas educativas que se han hecho, y que siguen vigentes, en el sistema de educación básica y media superior mexicano no han conseguido los resultados esperados, y las causas pueden ser muchas, como la permanencia de una historia tradicional en el aula, la falta de capacitación docente en la enseñanza del pensamiento histórico y la exclusión de la historia reciente de los programas de estudio.

Las investigaciones de Cerri \& Amézola (2010), inspiradas en el proyecto europeo Youth and History, indagaron el tema de la conciencia histórica en los jóvenes, vinculada estrechamente con sus actitudes políticas, en Brasil, Argentina y Uruguay. Los resultados subrayan que se continúa reproduciendo la noción de que las personas comunes no son importantes, dentro del razonamiento de que si lo fueran, se las consideraría objeto de interés. Las causas de esas divergencias están relacionadas con los patrones de enseñanza de la historia vivenciados por alumnos y profesores. Finalmente, el estudio de González \& Gárate (2017), sobre las perspectivas de los jóvenes acerca de diversos temas vinculados a la historia en cinco escuelas de la ciudad de Santiago de Chile, evidenció una alta valoración que otorgan los jóvenes al estudio de la historia y el reconocimiento de la sólida vinculación existente entre los problemas históricos y su propia vida, junto con una notoria presencia de prácticas y formas de aprendizaje de tipo tradicional.

\section{Método}

El objetivo principal de este artículo es analizar el contenido y el nivel cognitivo en exámenes de historia de estudiantes de colegios de públicos de Bogotá. Se utilizó un diseño metodológico mixto, que combinó la sistematización de datos cualitativos y cuantitativos del contenido de los exámenes.

\section{Variables}

El análisis del contenido de los exámenes fue orientado en función de la indagación de las siguientes variables: temáticas evaluadas, tipo y contenido del enunciado presente en la evaluación, formato de la evaluación, momento del proceso educativo en el que se hace la evaluación, tarea exigida a los estudiantes en los enunciados de cada pregunta y otra información presente en el texto del examen, como indicaciones y criterios de calidad. 


\section{Muestra}

Se trabajó con una muestra de 106 exámenes de ciencias sociales de educación primaria y secundaria en Bogotá (tabla 1).

Tabla 1

Muestra de exámenes analizados

\begin{tabular}{|c|c|c|}
\hline Institución & N. o de exámenes $^{\text {N.o }}$ de preguntas \\
\hline 1 & 5 & 28 \\
2 & 7 & 79 \\
3 & 12 & 183 \\
4 & 5 & 25 \\
5 & 5 & 46 \\
6 & 17 & 222 \\
7 & 6 & 27 \\
8 & 5 & 80 \\
9 & 6 & 34 \\
10 & 6 & 51 \\
11 & 18 & 199 \\
12 & 5 & 33 \\
13 & 10 & 99 \\
\hline Total & 106 & 1105 \\
\hline
\end{tabular}

Fuente: elaboración propia

Los exámenes incluidos en la muestra fueron aplicados entre febrero y agosto del año 2018 en trece instituciones educativas de Bogotá, como se muestra en la tabla 1.

\section{Recolección de los datos}

Tuvo dos etapas. En un primer momento se hizo una selección aleatoria de instituciones educativas de Bogotá de una lista disponible en la página web de la Secretaría de Educación del Distrito. A dichas instituciones se les envió una invitación para participar en el proyecto de investigación, previa explicación de sus objetivos y alcances. En la segunda etapa, aquellas que aceptaron participar en el proyecto aportaron los exámenes para que fueran analizados en el marco de la investigación. Se trabajó con evaluaciones parciales, finales y de recuperación. Algunos exámenes fueron entregados en forma digital, y los que fueron entregados en forma impresa se digitalizaron.

\section{Sistematización y análisis de datos}

El primer paso de la sistematización de la información fue la asignación de un código a cada institución y a cada examen. Posteriormente, se diseñó una matriz en Excel, en la que se consignaba la siguiente información 
de cada examen: curso; número de preguntas; formato del examen; tipo de evaluación; indicaciones para los estudiantes; la posibilidad de tener explicaciones del profesor y de sacar materiales de trabajo, como libros, cuadernos y guías durante el examen; las temáticas evaluadas; los criterios de calidad de la prueba; el enunciado de las preguntas, y los conocimientos y habilidades evaluadas.

Una vez finalizada la sistematización de los datos, estos fueron sometidos a un procesamiento cualitativo y a otro cuantitativo: los datos de contenido, es decir, los enunciados de las preguntas se codificaron con Nvivo, y se obtuvo un mapa jerárquico y una nube de palabras. El resto de la información fue sometida a un análisis cuantitativo en Stata, del cual se obtuvieron diagramas de cajas, con las cuales se pudo cuantificar las habilidades y los conocimientos evaluados en los exámenes.

Para comprobar la validez del instrumento para esta investigación, se ejecutaron dos acciones: una evaluación de expertos y una prueba piloto. La matriz, elaborada con base en la propuesta de Gómez, Rodríguez \& Miralles (2015), fue enviada a dos expertos en educación con experiencia en investigaciones sobre procesos de evaluación. Esta evaluación fue muy útil, porque el concepto del experto permitió reorientar las categorías planteadas inicialmente. El evaluador señaló que algunas categorías eran repetitivas y que otras podían generar confusión en la lectura de las preguntas y su sistematización. En consecuencia, la evaluación del experto permitió corregir la matriz de trabajo planteada inicialmente.

La aplicación de la prueba piloto se utilizó para ajustar el instrumento inicial (la matriz). Entre los ajustes más significativos, podemos destacar tres: la reducción de algunas categorías; el cambio en las opciones que podían generar ambigüedad y el reemplazo de algunos términos de la matriz, de manera que estos fueran familiares para todos los lectores del contenido de los exámenes a sistematizar, y la inclusión de un instructivo para realizar la lectura de los exámenes.

\section{Resultados}

El contenido de los exámenes indica que el eje articulador del currículo de historia es el tiempo. Como en otras investigaciones, la lectura de los enunciados de las preguntas sugiere que se sigue enseñando historia teniendo como eje un tiempo lineal que gira en torno a las épocas: prehistoria, época prehispánica, antigua, medieval, moderna y contemporánea.

El mapa jerárquico (figura 1) indica que la mayor parte de las preguntas formuladas en los exámenes son sobre las épocas moderna y contemporánea (el $16,9 \%$ de la primera y el $47,7 \%$ de la segunda). Entre las preguntas 
de este grupo se encuentran aquellas en las que se interroga a los estudiantes por el Renacimiento, el humanismo, las revoluciones burguesas, las guerras mundiales, los conflictos bélicos de las últimas décadas, el Imperialismo, la Conquista, Colonización, Independencia y la transición de dichos pueblos a la vida republicana y procesos actuales.

Figura 1

Mapa jerárquico

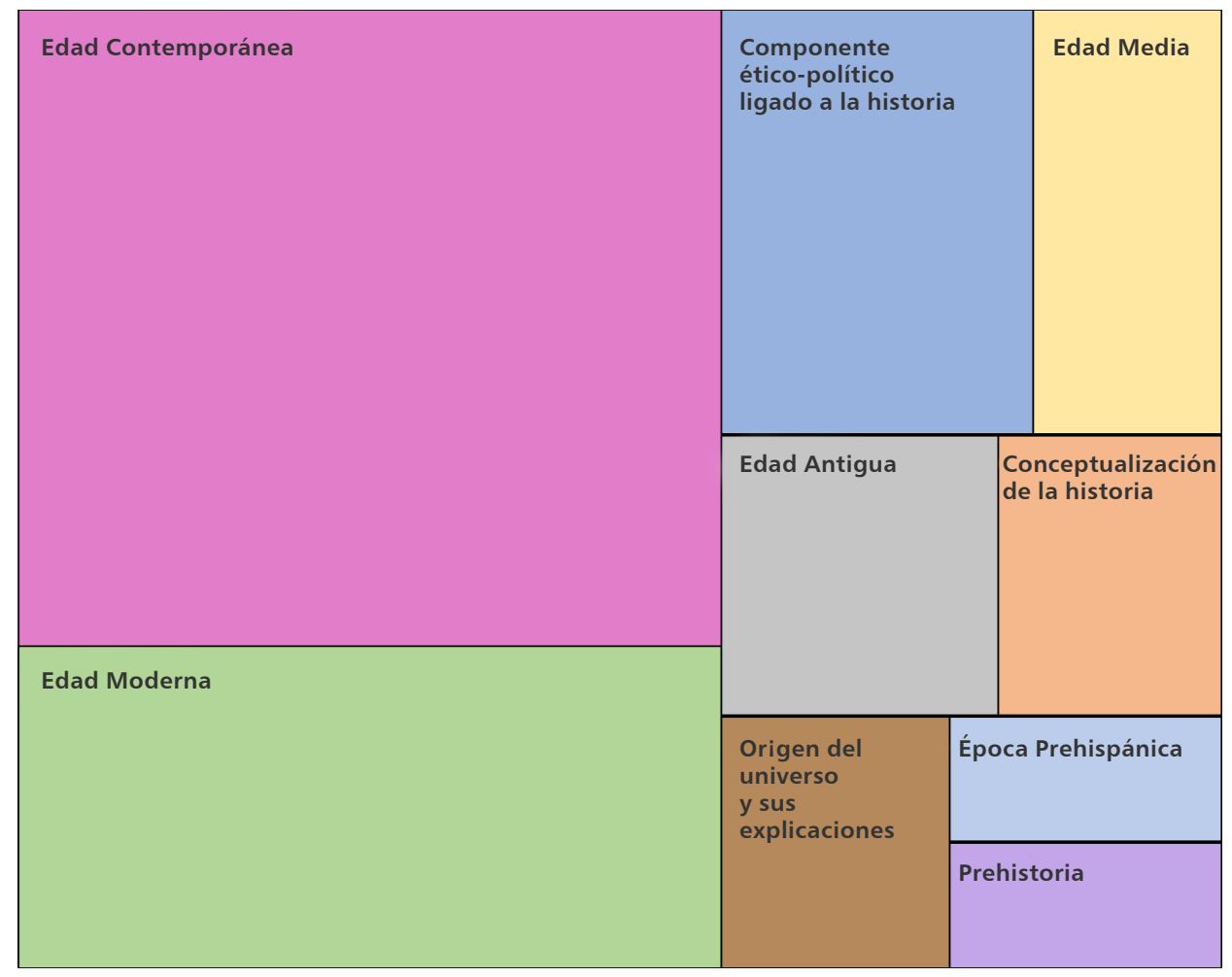

Fuente: elaboración propia

Un porcentaje muy similar (el 6,8\% y el 6,6\%, respectivamente) de las preguntas corresponden a la Edad Media y la historia antigua. Sobresalen preguntas sobre el Feudalismo, el papel de la Iglesia, las Cruzadas y las antiguas civilizaciones. El menor porcentaje de preguntas son sobre la época prehispánica (un 2,8\%) y sobre la prehistoria (el 1,9\%); en las primeras se interroga sobre civilizaciones y pueblos que poblaron América antes de la colonización europea y en las segundas, sobre las condiciones y las formas de organización social de los primeros pobladores de la tierra en diferentes contextos. Hay que destacar también que se identificó un grupo de preguntas, cuyo porcentaje equivale al 7,9\%, en las que se indaga sobre el componente ético-político ligado a la historia. Estas preguntas se detienen en temas como qué es la geopolítica, la Constitución, la estructura del Estado y las instituciones, la democracia, los mecanismos de participación y el 
origen e influencia de los organismos internacionales. En un 5,8\% se indaga por la conceptualización de la historia; en este componente se incluyen preguntas sobre el concepto de historia, su utilidad y el trabajo del historiador y su importancia. Finalmente, hay un 3,6\% en el que se indaga por el origen del universo y sus explicaciones.

Figura 2

Nube de palabras del contenido de las preguntas

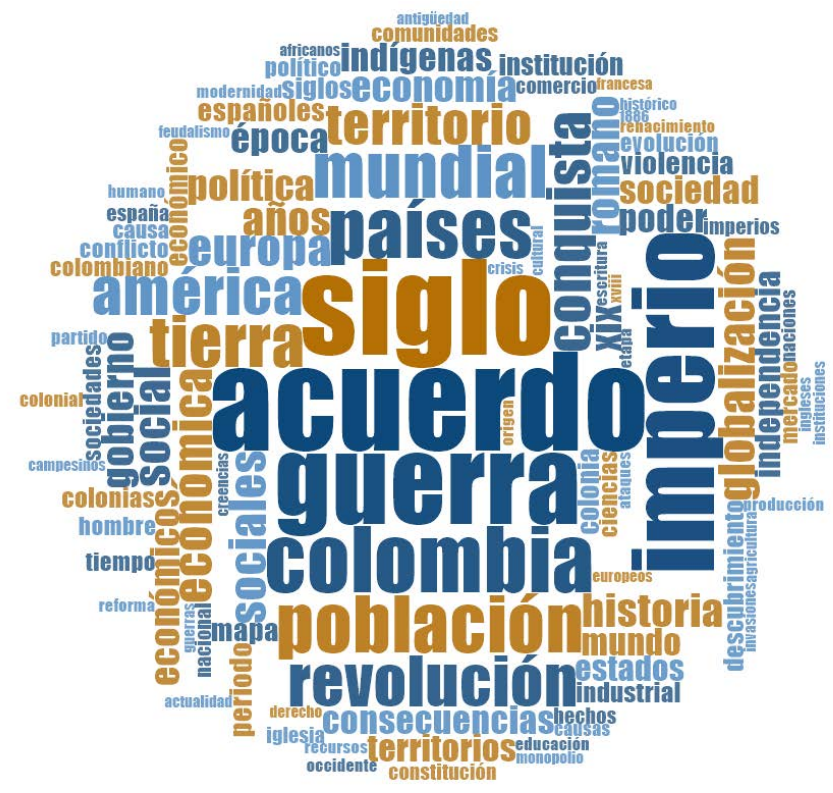

Fuente: elaboración propia

La densidad de las palabras de la nube (figura 2) ratifica los hallazgos reflejados en el mapa categorial sobre los contenidos: prevalecen los conceptos cronológicos y las temáticas de la época moderna y contemporánea. La importancia en los conceptos cronológicos queda demostrada en palabras como siglo, periodo, época, años, etapa y origen. La preponderancia de los temas de la época moderna y contemporánea se evidencian en palabras como guerra, imperio, globalización, conquista, independencia y revolución. Si bien en trabajos sobre la percepción de los estudiantes de la clase de las ciencias sociales, como el de Palacios (2018), los estudiantes expresan inconformidad porque los contenidos se centran en la enseñanza de la historia y la geografía de otros lugares y no de la realidad colombiana, la nube tiene la palabra Colombia como una de las preponderantes. También hay otras palabras que hacen referencia a otros lugares, como mundial, países, mundo, América, Europa, romano, españoles y España. La densidad de palabras relacionadas con el conflicto armado en Colombia, como acuerdo y guerra, pueden obedecer a que los maestros están abordando las temáticas 
de la actualidad del país relacionadas con el conflicto y el posacuerdo. Vale la pena destacar que las palabras reflejadas en la nube muestran que la historia política y la económica son las más trabajadas en clase, seguidas de la historia social y la historia cultural.

Figura 3

Distribución de preguntas según el tipo de prueba

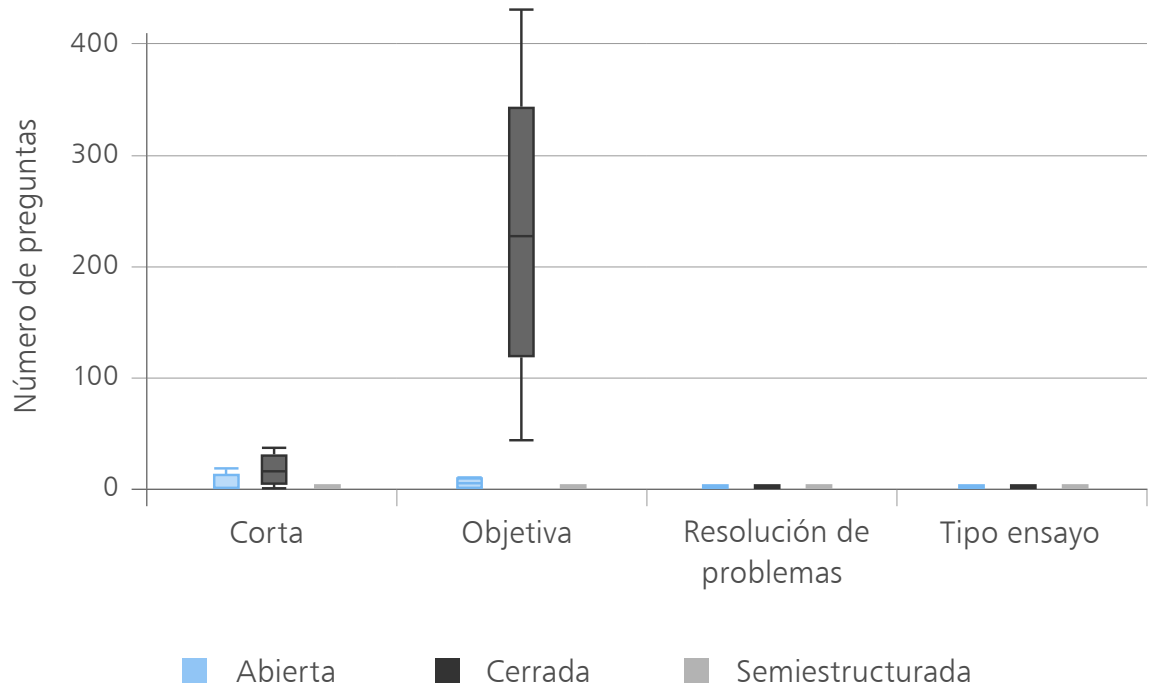

Fuente: elaboración propia, con base en la propuesta de Gómez et al. (2015)

Las evaluaciones se clasificaron, según su formato, en: evaluaciones de corta extensión, en las cuales se hace usualmente una o dos preguntas abiertas que los estudiantes deben desarrollar; evaluaciones objetivas, que tiene un contexto del cual surge la pregunta, o de tipo test, en las cuales se presenta al estudiante una información (texto, cuadro, mapa o dibujo) y posteriormente se formula una pregunta con una o varias opciones de respuesta. En las evaluaciones de resolución de problemas se presenta un contexto con una situación problemática concreta y se invita a los estudiantes a plantearle soluciones. Finalmente, en los exámenes tipo ensayo se invita a los estudiantes a escribir un texto sobre un enunciado o un tema en particular. Las preguntas de las evaluaciones anteriormente mencionadas fueron clasificadas en: abiertas, en las que el estudiante es libre de responder según su redacción y escritura; cerradas, en las cuales el estudiante debe responder según las opciones de respuesta, y semiestructuradas, en las que el estudiante debe completar o relacionar las opciones de respuesta.

De 1105 preguntas analizadas, el 8,14\% corresponden a preguntas cortas; dentro de esas preguntas cortas, el 6,1\% son preguntas cerradas y 
el 1,9\% son preguntas abiertas; no se encontraron preguntas semiestructuradas. Las pruebas objetivas son el $91 \%$ del total de las preguntas analizadas; dentro de las pruebas objetivas, las preguntas cerradas son el 87,4\%, mientras que las preguntas abiertas son el 2,2\% y las preguntas semiestructuradas el 1,2\%. Finalmente, las pruebas de resolución de problemas tienen preguntas de tipo ensayo, que en la muestra total arrojaron un porcentaje del $0,81 \%$.

Es importante aclarar que en la comunidad escolar de los colegios públicos se utilizan los espacios para evaluar como una oportunidad de preparación para la Prueba Saber $11^{\circ}$, las cuales tienen como característica la evaluación por competencias, o, dicho de otro modo, utilizar el conocimiento para resolver situaciones. Llama la atención la manera en que los maestros están elaborando las pruebas objetivas, que incluyen preguntas cerradas de opción única o múltiple, como las utilizadas en las pruebas Saber, pues estas usualmente no invitan a los estudiantes a resolver situaciones que involucran la aplicación o utilización de conocimientos previamente adquiridos.

Una de las razones que explica el uso generalizado de las evaluaciones objetivas es que se pueden calificar en un espacio de tiempo mucho más corto que otro tipo de evaluación, hecho importante si se tiene en cuenta el número de estudiantes que usualmente conforman un curso en ciudades como Bogotá.

Figura 4

Distribución de las habilidades cognitivas según el momento

en que se realiza la evaluación

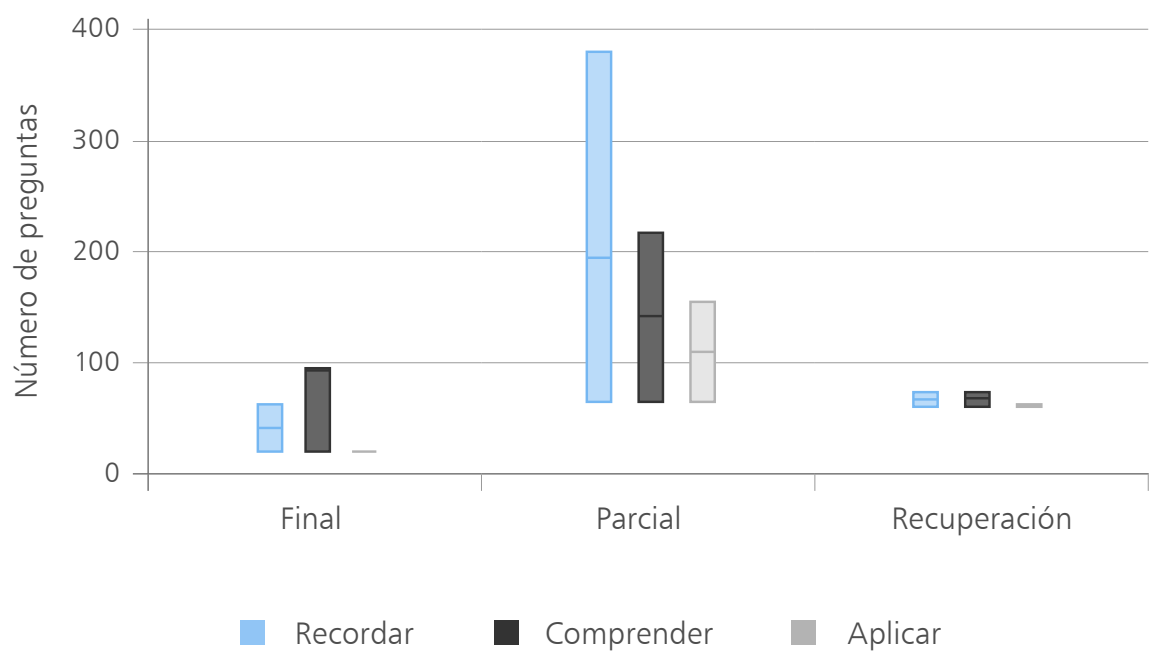

Fuente: elaboración propia, con base en la propuesta de Gómez et al. (2015) 
También se analizó cada uno de los exámenes según el momento del año escolar en que se aplica y, dentro de esta categoría, las habilidades de pensamiento histórico evaluadas: el 19,3\% son exámenes que se aplican para finalizar el año escolar; dentro de estos, el $7 \%$ evalúa la habilidad de recordar, el 11,5\% la habilidad de comprender y el 0,7\% la habilidad de aplicar. En los exámenes parciales, que corresponden al 76,7\% de la muestra, se evalúa en el 39,6\% la habilidad de recordar, en el 20,3\% la habilidad de comprender y en el 16,8\% la habilidad de aplicar. Finalmente, los exámenes de recuperación son el 3,9\% de la muestra, y allí se evalúan las habilidades de recordar y comprender (con el 1,5\% cada una) y la habilidad de aplicar (con el 0,9\%). No se encontraron dentro de la muestra entregada por los profesores e instituciones que participaron evaluaciones iniciales o diagnósticas.

Figura 5

Distribución de preguntas de tipo de prueba según el nivel cognitivo evaluado

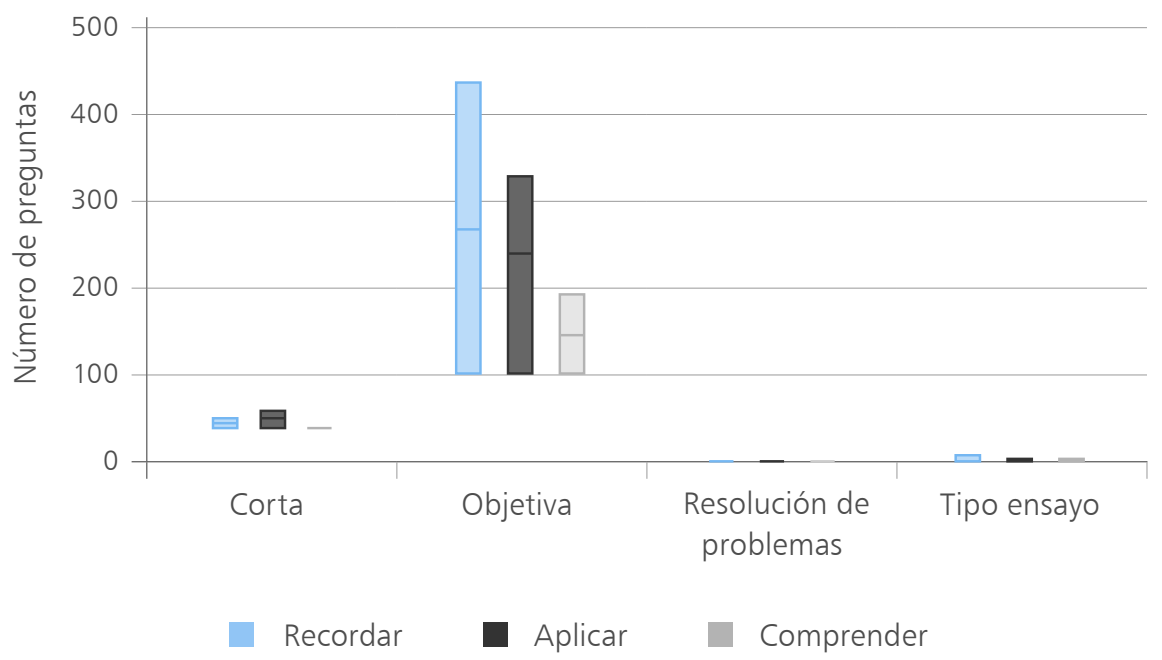

Fuente: elaboración propia, con base en la propuesta de Gómez et al. (2015)

En la muestra analizada, también se cruzan los datos de tipo examen con habilidad evaluada. Allí se encuentra que, dentro de las pruebas cortas, el 5,6\% de las preguntas evalúan la habilidad de recordar, el 7,1 \% la habilidad de comprender y el $4 \%$ la habilidad de aplicar. En las pruebas objetivas, el 34,8\% de las preguntas evalúan la habilidad de recordar, el 28,9\% la habilidad de comprender y el 18,2\% la habilidad de aplicar. Finalmente, las pruebas de resolución de problemas evalúan la habilidad de aplicar en un $0,084 \%$, y las pruebas tipo ensayo evalúan la habilidad de recordar en un $0,25 \%$, la habilidad de comprender en un 0,08\% y la habilidad de aplicar en el $0,04 \%$. 


\section{Discusión de los resultados y conclusiones}

Si bien el área de ciencias sociales cuenta con unos lineamientos curriculares que proponen abordar su enseñanza a través de ejes generadores, preguntas problematizadoras y ámbitos conceptuales (Ministerio de Educación Nacional de Colombia, 2002), y aunque en Bogotá se publicó un documento titulado Orientaciones curriculares para el campo del pensamiento histórico, de lineamientos la enseñanza de la historia a partir del pensamiento histórico (Secretaría de Educación Distrital, 2007), tanto el mapa jerárquico (figura 1) como la nube de palabras (figura 2) arrojan que se sigue trabajando bajo una concepción de historia lineal basada en épocas, de las cuales las más trabajadas son la moderna y la contemporánea, con contenidos de historia política e historia económica. Ejemplos de dichas preguntas son las siguientes:

8. En 1886 nuestro país recibió el nombre de República de Colombia, que se ha mantenido hasta la actualidad. Ese cambio se hizo en el año de: [...].

1. ¿Cuándo tuvo lugar la primera Revolución Industrial? [...].

10. ¿Cómo se llama el personaje que descubrió América? [...].

9. ¿Cuál es el nombre del dictador fascista?

Estas son algunas de las preguntas en las que se evidencia la persistencia de una historia lineal, en la que se evalúa la exposición de datos, como fechas, situaciones y personajes, como parte del ejercicio memorístico para "recordar" lo aprendido. Lo anterior pone en la mesa el debate sobre el tipo de enseñanza y el tipo de evaluación de las ciencias sociales y de la historia que en estos momentos se está trabajando en el aula de clase.

Por otra parte, los datos que se han presentado en este artículo a partir de la lectura de exámenes de historia en algunos colegios públicos de Bogotá (figuras 4 y 5) enriquecen el debate sobre la necesidad de repensar la enseñanza de la disciplina y aportan evidencias que permiten reforzar los hallazgos de otros estudios que han señalado que persisten actividades de aprendizaje e instrumentos de evaluación en los que principalmente se solicita a los estudiantes memorizar y recordar datos (Gómez, Molina \& Pagán, 2012; Monteagudo et al., 2015; Monteagudo \& Vera, 2017). Tal y como han establecido estos autores para el caso de España, los exámenes analizados indican que nos encontramos ante una instrucción que, además de ser muy elemental, privilegia los contenidos conceptuales e involucra muy poco los contenidos procedimentales.

La prevalencia numérica de las preguntas en las que se solicita a los estudiantes la actividad cognitiva de "recordar" indica que, si bien en la retórica de los maestros se han incorporado enfoques teóricos y metodológicos 
de pedagogías activas, como el constructivismo, esto no se refleja en el aula. Monteagudo \& Villa (2011) subrayan que una de las principales limitaciones de privilegiar el recuerdo es que se priva a los estudiantes de desarrollar otras habilidades cognitivas más complejas, que también son fundamentales en la escuela primaria y secundaria, como el "comprender y aplicar procedimientos y conceptos" (p. 62).

La escasez de preguntas en las que se solicita a los estudiantes que reflexionen o den solución a problemáticas de su vida cotidiana es evidente, pues solo se identificaron dos preguntas que ponen de manifiesto que los procesos de evaluación del aprendizaje reflejados en los 106 exámenes analizados incorporan solo en una mínima parte el reto de orientar a los alumnos en la "comprensión de los grandes problemas y situaciones sociales que estudiantes y maestros enfrentan y viven cotidianamente" (Ministerio de Educación Nacional de Colombia, 2006, p. 121). La falta de reflexión sobre los temas de la cotidianidad colombiana y de la realidad inmediata de los estudiantes preocupa, porque, como bien se enuncia tanto en los estándares como en los lineamientos de ciencias sociales, es fundamental que, en su paso por la educación primaria y secundaria, los niños y los jóvenes del país "asuman posturas éticas, que no necesariamente son homogéneas, sino que respetan el carácter plural y diverso de los seres humanos en procura de transformar las condiciones adversas que condicionan y limitan la dignidad humana (Ministerio de Educación Nacional de Colombia, 2006, p. 119).

La preponderancia de la exigencia de la habilidad de recordar, por encima de la habilidad de comprender y de aplicar, demuestra una tendencia a evaluar conceptos de primer orden (Gómez \& Miralles, 2017), que corresponde a las habilidades cognitivas exigidas en los exámenes analizados. Dichos exámenes muestran que mayoritariamente las preguntas exigen un dominio memorístico y la ausencia de procedimientos. Los resultados evidencian una evaluación que mide aprendizaje de contenidos y la ausencia de otras habilidades de pensamiento histórico, como la explicación causal, es decir, aplicar y explicar los conocimientos en una situación dada y utilizar las pruebas, argumentos y conclusiones basados en las pruebas.

En este sentido, el pequeño número de preguntas de tipo ensayo plantea la necesidad de desarrollar la competencia narrativa, la cual es definida por Rüsen (2007) "como la habilidad para desarrollar procedimientos que dan sentido pasado" (p. 26). Según Salazar (2006, citado por Santisteban, 2010),

el valor de la narrativa histórica en la educación se encuentra en el hecho de que es una representación de acontecimientos reales, organizados 
en una trama que responde a un problema planteado por la realidad, en la cual se muestra la experiencia humana y no solo conceptos abstractos (p. 44).

Otro aspecto de los resultados del artículo en el que hay que detenerse es la proporción de exámenes según el momento en que se presenta la prueba. Llamó la atención la inexistencia de exámenes de inicio o diagnósticos, sobre todo si desde las propuestas constructivistas, la enseñanza para la comprensión y la pedagogía basada en problemas se ha resaltado la pertinencia de considerar los conocimientos previos de los estudiantes e involucrarlos en sus procesos de aprendizaje. Las investigaciones de Pazzani (1991), Verdugo (1996) y Alvermann \& Hynd (1989) han evidenciado que los grupos de estudiantes en los que se aplicó una prueba diagnóstica y los profesores que utilizaron los resultados para orientar la enseñanza obtuvieron mejores resultados en los exámenes finales.

El hecho de que el 76,7\% de los exámenes sean pruebas parciales de final de bimestre sería un indicador de que el principal propósito de los exámenes es darles una calificación a los alumnos. Este argumento es ratificado tanto por la ausencia de pruebas diagnósticas, que ya señalamos, como por el porcentaje de pruebas de recuperación, que representan tan solo el 3,9\% de las analizadas. Si se tiene en cuenta que no hay evidencias de exámenes de diagnóstico, que hay un número pequeño de actividades de recuperación y que la mayor parte de los exámenes son de final de bimestre, podemos decir que la evaluación en ciencias sociales sigue siendo más sumativa que formativa. Llama la atención el bajo porcentaje de exámenes de recuperación, porque este puede ser un espacio idóneo para retroalimentar, potencializar lo aprendido y aclarar dudas del proceso de adquisición de habilidades, de allí que Soler (2007) Ilame la atención sobre la importancia que tienen los exámenes y las actividades de recuperación, pues estas podrían convertirse en un escenario para que los alumnos que no han logrado superar ciertos objetivos de los distintos niveles realicen actividades y evaluaciones con estrategias diferentes a aquellas primeras que hicieron que el objetivo no se consiguiera, para evitar que se produzcan estancamientos y que puedan conseguir lo que no han logrado antes.

La clara tendencia a incluir en los exámenes preguntas objetivas o de selección múltiple parece estar relacionada con el deseo de los maestros de dos cosas: una, preparar a los estudiantes para la Prueba Saber de Ciencias Sociales, aplicada por el Estado, y, dos, mejorar los resultados en dichas pruebas, en las que 24 de las 25 preguntas son de selección múltiple y solo una es abierta. Dicha tendencia evidencia por los menos dos tensiones: la que plantea la discusión sobre la pertinencia de las preguntas de selección 
múltiple para evaluar conocimientos y habilidades en el área de ciencias sociales y la que cuestiona la preponderancia que se le viene dando a las pruebas estandarizadas como indicadores para medir la calidad de la educación.

A la pregunta por la conveniencia de evaluar conocimientos y habilidades del área de ciencias sociales, como los conceptos, los procedimientos y las actitudes, con preguntas de selección múltiple, o de respuestas cortas, restringidas y limitadas, planteadas por autores como Palacios (2017), el Instituto Colombiano para la Evaluación de la Educación \& Ministerio de Educación Nacional (2013), en los documentos que orientan la interpretación de los resultados de las pruebas Saber, han sostenido que, aunque reconocen que los resultados obtenidos mediante preguntas cerradas siempre conllevan a imprecisiones derivadas de las respuestas dadas al azar y que hay una probabilidad relativamente grande de obtener una respuesta correcta mediante una selección aleatoria, defienden la preponderancia de dichas preguntas, apoyados en las pruebas de confiabilidad y validez a que son sometidas antes de su aplicación.

En cuanto a la segunda tensión, el uso masivo de las preguntas de selección múltiple parece estar relacionado con la importancia que han ganado las pruebas Saber en los últimos años. Además de ser uno de los indicadores para medir la calidad de la educación, obtener altos puntajes sirve para hacerse acreedor de becas, bonificaciones económicas y condecoraciones. Lo anterior no tendría ningún reparo, si no fuera porque el afán de obtener buenos resultados induce a algunos profesores a condicionar la acción en el aula, a seleccionar los contenidos y a orientar el proceso de evaluación para obtener buenos resultados en las pruebas externas (Sáiz, 2013; Souto \& Claudino, 2009).

Los testimonios aportados por estudiantes de último grado de secundaria sobre sus percepciones de la clase de ciencias sociales, en un trabajo publicado por Palacios (2018), muestran que buena parte de las actividades del aula de dicho curso son dedicadas a entrenar a los estudiantes para dar respuestas a test similares a la Prueba Saber para que les "vaya bien", y para contestar correctamente un examen, lo que no solamente desdibuja completamente el propósito y orientación del área, sino que desaprovecha las capacidades de los estudiantes para construir conocimientos y desarrollar habilidades útiles para la vida.

Una alternativa a las problemáticas analizadas en el artículo es reemplazar la planeación de clases desde unidades simples o temas, que generalmente conllevan el uso del examen como el principal o único instrumento de evaluación, por planeaciones desde unidades complejas, como el trabajo por proyectos o unidades didácticas, o el aprendizaje basado en problemas. 
Instituciones educativas donde han adoptado la planeación de las clases desde unidades complejas y a partir de una problemática, bien sea social, política o medioambiental, proponen a sus estudiantes productos y metas de aprendizaje que involucran diferentes asignaturas. Desde estas perspectivas no solo se promueve una formación más enriquecedora, sino que se adoptan instrumentos de evaluación diversos, como portafolios, experiencias de trabajo de campo y reflexiones críticas, que generan mejores formas de retroalimentar los aprendizajes de los estudiantes y de reflexionar la práctica docente.

Otra alternativa sería el replanteamiento de las formas de evaluar por parte del Estado a los estudiantes de los últimos grados. Aunque esto plantee la necesidad de una inversión más alta en recursos económicos, personal y tiempo, es necesario, sobre todo si, como anuncia el propio Estado, se le está apuntando a la formación de estudiantes con capacidades de abstracción y de solución de problemas, relacionados con su entorno y con el mundo. Lo anterior implica no solamente un claro compromiso político, sino también una recomposición estructural del sistema de evaluación de la educación en el país.

\section{Agradecimientos y aclaraciones}

Agradecimientos a la Vicerrectoría de Investigaciones y a la Facultad de Educación de la Universidad de los Andes, Colombia, y a las instituciones educativas participantes en el proyecto.

\section{Sobre los autores}

Nancy Palacios-Mena es licenciada en Ciencias Sociales de la Universidad del Valle, Colombia, y magíster en Sociología de la misma universidad. Es estudiante del Doctorado en Ciencias Sociales, Niñez y Juventud del Cinde-Universidad de Manizales, Colombia. Profesora de la Facultad de Educación de la Universidad de los Andes, Colombia.

Luz Yehimy Chaves-Contreras es licenciada en Ciencias Sociales de la Universidad Distrital Francisco José de Caldas, Colombia. Es magíster en Historia de la Universidad Nacional de Colombia. Docente de la Secretaría de Educación de Bogotá, Colombia.

William Alfonso Martin-Moreno es licenciado en Educación Básica con Énfasis en Ciencias Sociales de la Universidad Distrital Francisco José de Caldas, Colombia, y magíster en Asuntos Internacionales de la Universidad Externado de Colombia. Docente de la Secretaría de Educación de Bogotá, Colombia. 


\section{Referencias}

Alfageme, M. \& Miralles, P. (2009). Instrumentos de evaluación para centrar nuestra enseñanza en el aprendizaje de los estudiantes. Íber. Didácticas de las Ciencias Sociales, Geografía e Historia, 60, 8-20. Recuperado de https:// www.researchgate.net/publication/288879264_Instrumentos_de_evalua cion_para_centrar_nuestra_ensenanza_en_el_aprendizaje_de_los_estu diantes

Alvermann, D. \& Hynd, C. (1989). Effects of prior knowledge activation modes and text structure on nonscience majors' comprehension of physics. Journal of Educational Research, 83, 97-102.

Asensio, M., Carretero, M. \& Pozo, J. (1986). La comprensión de la historia. Pensamiento relativista. Cuadernos de Pedagogía, 33, 24-27.

Calatayud, M. (2000). La supremacía del examen: la evaluación como examen, su uso y abuso, aún en la educación primaria. Bordón. Revista de Pedagogía, 52(2), 165-178.

Carrretero, M. (1993). Constructivismo, desarrollo cognitivo y educación. Madrid: Edelvives.

Carretero, M. \& Limón, M. (1993). Aportes de la psicología cognitiva y la instrucción a la enseñanza de la historia y las ciencias sociales. Revista Infancia y Aprendizaje, 62(63), 153-167.

Carretero, M. \& Montanero, M. (2008). Enseñanza y aprendizaje de la historia: aspectos cognitivos y culturales. Revista Cultura y Educación, 20(2), 133-142. Recuperado de http://www.ub.edu/histodidactica/images/docu mentos/pdf/ensenanza_aprendizaje_historia.pdf

Carretero, M., Pozo, J. \& Asensio, M. (Eds.). (1989). La enseñanza de las ciencias sociales. Madrid: Visor.

Carretero, M. \& Voss, J. (Comps.). (2004). Aprender y pensar la historia. Buenos Aires: Amorrortu.

Castillo, M. (2004). La evaluación por competencias y sus implicaciones pedagógicas. Revista Enunciación, 9(1), 68-78. doi: https://doi.org/10.144 83/22486798.2491

Cerri, L. \& Amézola, G. (2010). El estudio empírico de la conciencia histórica en jóvenes de Brasil, Argentina y Uruguay. Revista Didáctica de las Ciencias Experimentales y Sociales, 24, 4-23. Recuperado de https://core.ac.uk/ download/pdf/71003590.pdf

Cooper, H. (2013). Teaching History Creatively. Londres: Routledge

Domínguez, J. (2015). Pensamiento histórico y evaluación de competencias. Barcelona: Graó.

Éthier, M., Demers, S. \& Lefrançois, D. (2010). Las investigaciones en didáctica sobre el desarrollo del pensamiento histórico en la enseñanza primaria. Una panorámica de la literatura publicada en francés e inglés desde el año 1990. Revista Enseñanza de las Ciencias Sociales, 9, 61-74. Recuperado de https:// www.redalyc.org/pdf/3241/324127609007.pdf

Fuster, C. (2017). ¿Estamos preparados para evaluar el conocimiento histórico? Revista GeocritiQ, 278, 1-5. 
Gómez, C. \& Miralles, P. (2013). Los contenidos de ciencias sociales y las capacidades cognitivas en los exámenes de tercer ciclo de educación primaria. ¿Una evaluación en competencias? Revista Complutense de Educación, 24(1), 91-121. doi: 10.5209/rev_RCED. 2013. v24. n1.41193

Gómez, C. \& Miralles, P. (2017). Los espejos de Clío. Usos y abusos de la historia en el ámbito escolar. Madrid: Silex.

Gómez, C., Miralles, P. \& Molina, S. (2015). Evaluación, competencia histórica y educación ciudadana. Revista de Estudios Sociales, 52, 9-14. Recuperado de https://www.redalyc.org/pdf/815/81538634001.pdf

Gómez, C., Molina, S. \& Pagán, B. (2012). Los manuales de ciencias sociales y la enseñanza de la historia del arte en $2 .^{\circ}$ de ESO. Ensayos, Revista de la Facultad de Educación de Albacete, 27, 69-88. Recuperado de https://dialnet. unirioja.es/servlet/articulo?codigo $=4202766$

Gómez, C., Rodríguez, R. \& Miralles P. (2015). La enseñanza de la historia en educación primaria y la construcción de una narrativa nacional. Un estudio sobre exámenes y libros de texto en España. Revista Perfiles Educativos, 37(150), 20-38. Recuperado de http://www.scielo.org.mx/scielo. php?pid=S0185-26982015000400002\&script=sci_abstract

Gómez-Carrasco, C. J., Miralles-Martínez, P. \& Chapman, A. (2017). Los procedimientos de evaluación en la clase de Historia. Un análisis comparativo a través de las opiniones de los docentes en formación en España e Inglaterra. Revista Electrónica Interuniversitaria de Formación del Profesorado, 20(2), 45-61.

González, F. \& Gárate, C. (2017). El aprendizaje histórico en la educación secundaria. Jóvenes chilenos y conciencia histórica. Diálogo Andino, 53, 73-85. Recuperado de http://dx.doi.org/10.4067/S0719-26812017000200073

Guimera, C. \& Carretero, M. (1992). Práctica docente y pensamiento del profesor de secundaria. En A. Estebaranz \& V. Sánchez (Eds.), Pensamiento de profesores y desarrollo profesional (pp. 301-308). Sevilla: Secretariado de Publicaciones Universidad de Sevilla.

Instituto Colombiano para la Evaluación de la Educación (Icfes) \& Ministerio de Educación Nacional (MEN). (2013). Sistema de evaluación y estandarización. Alineación del examen Saber 11. Bogotá: Autor. Recuperado de http://www. paidagogos.co/nuevo_saber11/ejemplos_socialesyciudadanas.pdf

López, R. (1997). La práctica de la evaluación aplicada al área de ciencias sociales en la enseñanza secundaria. En H. Salmerón (Ed.), Evaluación educativa. Teoría, metodología y aplicaciones en áreas de conocimiento (pp. 371-397). Granada: Grupo Editorial Universitario.

Lucero, M. \& Montanero, M. (2008). La explicación multicausal en el aula de historia. Tres experiencias de asesoramiento psicopedagógico. Revista Infancia y Aprendizaje, 31(1), 45-65. Recuperado de http://hdl.handle. net/11162/76096

Mainer, J. (2002). Pensar históricamente el examen, para problematizar su presente. Notas para una sociogénesis del examen. Gerónimo de Uztáriz, 1718, 107-135. Recuperado de http://amarauna.org/uztariz/pdf/artikuluak/ aldizkaria1707.pdf 
Martineau, R. (1999). L'histoire à l'école, matière à penser. París: L'Harmattan.

Merchán, F. (2005). La enseñanza, examen y control: profesores y alumnos en la clase de historia. Barcelona: Octaedro.

Merchán, F. J. (2009). Hacer extraño lo habitual. Microsociología del examen en la clase de historia. Íber. Didáctica de las Ciencias Sociales, Geografía e Historia, 60, 21-34.

Miralles, P., Molina, S. \& Santisteban, A. (2011). La evaluación en el proceso de enseñanza y aprendizaje de las ciencias sociales. 2 vols. Murcia: Asociación Universitaria del Profesorado de Didácticas de las Ciencias Sociales.

Ministerio de Educación Nacional (MEN). (15 de febrero de 2002). Decreto 230. Por el cual se dictan normas en materia de currículo, evaluación y promoción de los educandos y evaluación institucional.

Ministerio de Educación Nacional (MEN). (16 de abril de 2002). Decreto 1290. Por el cual se reglamenta la evaluación del aprendizaje y promoción de los estudiantes de los niveles de educación básica y media. Recuperado de https://www.mineducacion.gov.co/1621/articles-187765_archivo_pdf_decre to_1290.pdf

Ministerio de Educación Nacional Colombia (MEN). (2002). Lineamientos curriculares para el área de ciencias sociales. Bogotá: Autor. Recuperado de https:// www.mineducacion.gov.co/1621/articles-89869_archivo_pdf.pdf

Ministerio de Educación Nacional de Colombia (MEN). (2006). Estándares básicos de competencias en lenguaje, matemáticas, ciencias y ciudadanas. Bogotá: Autor.

Molina, S. \& Calderón, D. (2009). Los criterios de evaluación de Geografía e Historia en segundo ciclo de la ESO: análisis curricular comparativo. Didáctica de las Ciencias Experimentales y Sociales, 23, 37-60. Recuperado de https:// www.google.com/url?sa $=t \& r c t=j \& q=\& e s r c=s \&$ source $=$ web $\& c d=1 \& v e d=$ 2ahUKEwi_z62notHIAhWHxVkKHbl_B7UQFjAAegQIARAC\&url=https\%3A \%2F\%2Fdialnet.unirioja.es\%2Fdescarga\%2Farticulo\%2F3082101.pdf\&usg= AOvVaw3EEUuslz1aJ9L5rMnQKoeV

Montanero, M. (2001). La enseñanza de estrategias de razonamiento en el área de ciencias sociales. Íber. Didácticas de las Ciencias Sociales, 29, 95-105.

Monteagudo, J., Molina, S. \& Miralles, P. (2015). Opiniones sobre evaluación de los profesores de segundo ciclo de ESO de Geografía e Historia de España. El caso de la región de Murcia. Revista Mexicana de Investigación Educativa, 20(66), 737-761. Recuperado de http://www.scielo.org.mx/scielo. php?script=sci_arttext\&pid=S1405-66662015000300004

Monteagudo, J. \& Vera, M. (2017). Qué aprenden los alumnos de ESO sobre historia del arte. Un análisis de la legislación y los exámenes en el caso de la región de Murcia. Revista Educatio Siglo XXI, 35(3), 229-254. doi: https:// doi.org/10.6018/j/308981

Monteagudo, J. \& Villa, J. L. (2011). La evaluación de las competencias básicas en la materia de Historia en $4 .^{\circ}$ de ESO en la región de Murcia. En P. Miralles, S. Molina \& A. Santisteban (Eds.), La evaluación en el proceso de enseñanza y aprendizaje de las ciencias sociales (pp. 317-325). Vol. I. Murcia: Asociación Universitaria de Profesorado de Didáctica de las Ciencias Sociales. 
Monteagudo, J., Villa, J. \& Miralles, P. (2015). Las prácticas de evaluación en las materias de ciencias sociales de ESO en la región de Murcia según la opinión de los inspectores de educación. Revista Didácticas de las Ciencias Experimentales y Sociales, 29, 61-88. Recuperado de https://www.researchgate. net/profile/Pedro_Miralles_Martinez/publication/289584434_Las_practi cas_de_evaluacion_en_las_materias_de_Ciencias_Sociales_de_ESO_en_la_ Region_de_Murcia_segun_la_opinion_de_los_inspectores_de_Educacion/ links/569392d008aec14fa55eb455/Las-practicas-de-evaluacion-en-lasmaterias-de-Ciencias-Sociales-de-ESO-en-la-Region-de-Murcia-segun-laopinion-de-los-inspectores-de-Educacion.pdf

Pagès, J. (2009). El desarrollo del pensamiento histórico como requisito para la formación democrática. Revista Reseñas de Enseñanza de la Historia, 7, 69-91.

Palacios, N. (2017). La prueba de ciencias sociales en Colombia y su utilidad pedagógica para los maestros. Revista Didáctica de las Ciencias Sociales y Experimentales, 33, 21-22. doi: 10.7203/DCES.33.10833

Palacios, N. (2018). Perceptions of secondary school students of the social science class: A study in three Colombian institutions. International Journal of Instruction, 11(4), 353-374. Recuperado de https://doi.org/10.12973/ iji.2018.11423a

Plá, S. (2012). La enseñanza de la historia como objeto de investigación. Revista Secuencia, 84, 163-84. Recuperado de https://www.redalyc.org/ pdf/3191/319128360007.pdf

Plá, S. \& Pagès. J. (2014). La investigación en la enseñanza de la historia en América Latina. México D. F.: Universidad Pedagógica Nacional (UPN) y Bonilla Artigas.

Plá, S. \& Perez, M. (2013). Pensar históricamente sobre el pasado reciente en México. Revista Clío \& Asociados, 17, 27-55. Recuperado de http://www. memoria.fahce.unlp.edu.ar/art_revistas/pr.6208/pr.6208.pdf

Pazzani, M. (1991). Influence of prior knowledge on concept acquisition: Experimental and computational results. Journal of Experimental Psychology: Learning, Memory, and Cognition, 17(3), 416-432.

Pérez, M. C. (1995). Historia del arte. Materiales didácticos. Bachillerato. Madrid: MEC.

Pérez, V. (2015). Del examen a la evaluación pasando por el paradigma del emprendedor. Evaluar ipara qué?, ¿para quién es? Revista Con-ciencia Social: Anuario de Didáctica de la Geografía, la Historia y las Ciencias Sociales, 19, 145-152. Recuperado de https://dialnet.unirioja.es/servlet/ articulo?codigo $=5566729$

Pozo, J. \& Carretero, M. (1989). Las explicaciones causales de expertos y novatos en historia. En M. Carretero, J. Pozo \& M. Asensio (Comps.), La enseñanza de las ciencias sociales (pp.139-163). Madrid: Visor.

Prado, V. \& Medina, A. (2014). Características de la enseñanza y la evaluación de los aprendizajes en educación básica primaria. Papeles, 11, 20-35. Recuperado de http://csifesvr.uan.edu.co/index.php/papeles/article/view/394

Prats, J. (2016). Combates de la historia en educación. Enseñanza de las ciencias sociales. Revista Investigación, 15, 145-153. Recuperado de https://www. raco.cat/index.php/EnsenanzaCS/article/view/316818 
Ravela, P. (2010). ¿Qué pueden aportar las evaluaciones estandarizadas a la evaluación en el aula? Programa de Promoción de la Reforma Educativa en América Latina y el Caribe. Preal, Serie Documentos, n. ${ }^{\circ}$ 47. Santiago de Chile: Cinde. Recuperado de https://www.researchgate.net/publica tion/48139769_Que_pueden_aportar_las_evaluaciones_estandarizadas_a_ la_evaluacion_en_el_aula

Rincón, G. (2008). La enseñanza significativa de las ciencias sociales en grado décimo (tesis de pregrado). Universidad Nacional Abierta y a Distancia, Bogotá, Colombia.

Rojas, R. (2014). Competencias en lenguaje: relaciones entre la escuela y las Pruebas de Estado. Estudios sobre la calidad de la educación en Colombia. Bogotá: Icfes.

Rüsen, J. (2007). How to make sense of the past-salient issues of metahistory. TD: The Journal of Transdisciplinary Research in Southern Africa, 3(1), 169-221.

Sáiz, J. (2013). Alfabetización histórica y competencias básicas en libros de texto de historia y en aprendizaje de los estudiantes. Revista Didácticas de las Ciencias Experimentales y Sociales, 27, 4366. Recuperado de https://ojs. uv.es/index.php/dces/article/view/2648

Sáiz, J. (2014). Fuentes históricas y libros de texto en secundaria: una oportunidad perdida para enseñar competencias de pensamiento histórico. Ensayos. Revista de la Facultad de Educación de Albacete, 29(1), 83-99.

Sáiz, J. \& López-Facal, R. (2014). Competencias y narrativas históricas: el pensamiento histórico de estudiantes y futuros profesores españoles de educación secundaria. Revista de Estudios Sociales, 52, 87-101. doi: 10.7440/ res52.2015.06

Santisteban, A. (2010). La formación de competencias de pensamiento histórico. Revista Clío \& Asociados, 14, 34-56. Recuperado de https://www.clio.fahce. unlp.edu.ar/article/view/clion14a03

Santos, M. (1993). La evaluación: un proceso dialógico, comprensión y mejora. Málaga: Aljibe.

Secretaría de Educación Distrital (SED). (2007). Orientaciones curriculares para el campo de pensamiento histórico. Bogotá: Autor. Recuperado de https:// repositoriosed.educacionbogota.edu.co/handle/001/896

Seixas, P. \& Morton, T. (2013). The Big Six Historical Thinking Concepts. Toronto: Nelson College Indigenous.

Shemilt, D. (1983). The devil is locomotive. Journal History and Theory, 22, 1-18.

Soler, E. (2007). Estrategias de recuperación escolar individualizada. Madrid: Perolibros.

Souto, X. \& Claudino, S. (2009). Exames de Geografia, programas e inovação didáctica. En A Inteligência Geográfica na Educação do Século XXI (pp. 21-30). Lisboa, APROFGEO/IGOT-UL/GDG-AGE.

Souto, X., Fuster, C. \& Sáiz, J. (2014). Un camino de ida y vuelta: reválidas y selectividad en las rutinas escolares de la enseñanza de Geografía e Historia. En J. Pagés y A. Santisteban (Eds.), Una mirada al pasado y un proyecto de futuro. Investigación e innovación en didáctica de las ciencias sociales, vol. 2. Barcelona: AUPDCS y Universitat Autónoma Barcelona. 
Trepat, C. (2012). La evaluación de los aprendizajes de historia y geografía en la enseñanza secundaria. Las pruebas de ensayo abierto. Íber. Didáctica de las Ciencias Sociales, Geografía e Historia, 70, 87-97.

Valls, R. \& López-Facal, R. (2011). ¿Un nuevo paradigma para la enseñanza de la historia? Los problemas reales y las polémicas interesadas al respecto en España y en el contexto del mundo occidental. Enseñanza de las ciencias sociales. Revista de Investigación, 10, 75-86. Recuperado de https://dialnet. unirioja.es/servlet/articulo?codigo $=4023128$

VanSledright, B. (2014). Assessing Historical Thinking and Understanding. Innovation Design for New Standards. Nueva York: Routledge.

Vera, M. I., Moreno, J. R. \& Torres, A. (2014). La enseñanza de la Historia de España: la percepción de los estudiantes en torno a las características de los docentes y su metodología. En N. Martínez (Dir.), La historia de España en los recuerdos escolares (pp. 179-210). Valencia: Nau Llibres.

Verdugo, A. (1996). Evaluación curricular: una guía para la intervención psicopedagógica. Salamanca: Siglo XXI. 